Research Article

\title{
Pattern Synthesis for Dual-Polarized Conformal Arrays via Iterative Convex Optimization
}

\author{
Chao Liu (D), Chuan Li, and Bo Yang \\ Dept. of Communication Engineering, Hefei University of Technology, Hefei, Anhui 230009, China \\ Correspondence should be addressed to Chao Liu; disneyl@hfut.edu.cn
}

Received 30 September 2020; Revised 19 March 2021; Accepted 22 March 2021; Published 27 April 2021

Academic Editor: Raffaele Solimene

Copyright (c) 2021 Chao Liu et al. This is an open access article distributed under the Creative Commons Attribution License, which permits unrestricted use, distribution, and reproduction in any medium, provided the original work is properly cited.

\begin{abstract}
An iterative convex optimization (ICO) algorithm is proposed to solve pattern synthesis problem under the framework of dualpolarized conformal arrays in this paper. The subproblems of shaping main lobe, optimizing side lobe, and suppressing crosspolarization component are summarized as a joint optimization problem. To solve this problem, the nonconvex constraint about main lobe is rewritten as a convex constraint, which will bring error. And an auxiliary phase function is introduced to correct this error alternatively. Due to the deviation between auxiliary phase and real phase of pattern function, a method minimizing the peak of the synthesis error over observation angles is effectively applied to further improve the performance of the method. Numerical examples show good pattern synthesis ability and convergence performance of the ICO method.
\end{abstract}

\section{Introduction}

Benefiting from their incomparable flexibility, conformal arrays have been widely applied to communication systems and aircrafts design, etc [1]. Compared with traditional planar arrays, the inconsistency of the elements' pattern and polarization feature brings enormous challenges to the pattern synthesis problem for conformal arrays.

The main purpose of conformal array pattern synthesis problem is to obtain desired pattern through optimizing the array weight vector and polarization information. Intelligence optimization algorithms such as genetic algorithm (GA) [2] and simulated annealing [3] are commonly used to solve this problem. However, these articles ignore optimization for cross-polarization component. In [4], the particle swarm optimization (PSO) method is applied to synthesize the elliptical and linear polarization patterns for dual-polarized array with shaped main lobe and low side lobe and crosspolarization level. In [5], an improved differential evolution algorithm is proposed to achieve lower side lobe levels and suppress the cross-polarization. Nevertheless, these stochastic optimization methods are computationally intensive because of their randomness in global optimization.
Other convenient and flexible methods are also utilized to synthesize polarization patterns. In $[6,7]$, the iterative least-squares method is used to achieve desired main lobe with polarization optimization. In [8], based on the adaptive theory, a numerical pattern synthesis method is proposed, which abandons the traditional method of applying virtual interference but controls the co-polarization and crosspolarization components by two weighting functions. In [9], an enhanced alternate projection algorithm is proposed to synthesize practical conformal array with mutual coupling corrected. In [10], a fast 3D synthesis method is proposed to synthesize copolarized and cross-polarized patterns with the consideration of dynamic range ratio of excitations. Although the above algorithms have a fast synthesis speed, the solution is easy to fall into the local optimal value.

The convex optimization algorithm can simultaneously optimize multiple problems expressed as a series of linear and convex quadratic constraints and can obtain the global optimal solution. Thus, it is also applied to the pattern synthesis problem. In [11], convex optimization starts to be really used for pattern synthesis with interior-point methods. In [12], the authors linearize the nonconvex constraints and divide the power pattern optimization 
problem into some subproblems, which can be solved by iterative second-order cone programming (SOCP). In $[13,14]$, it is studied as a convex programming problem to jointly synthesize the polarization and spatial power pattern. In [15], the constraints about constant modulus and main lobe are relaxed as convex constraints to solve. In [16], the authors use semidefinite relaxation to solve the nonconvex constraint to shape beam and control side lobe and crosspolarization. Compressive sensing (CS) theory and convex relaxation are used together to solve sparse conformal array synthesis problem, as described in [17]. It should be noted that the dual-polarized conformal arrays have advantages for the polarization information processing due to the two individual feed points of each dual-polarized antenna. In [18], a dualpolarized patterns optimization problem is formulated as a convex form, and related parameters determination method is presented. However, it lacks effective verification of the ability about main lobe shaping. It is worth studying pattern synthesis problem for dual-polarized conformal arrays.

This paper presents an iterative convex optimization (ICO) algorithm to synthesize pattern for dual-polarized conformal arrays. Main lobe shaping, side lobe optimization, and crosspolarization component suppression are formulated as a joint optimization problem. In the process of finding solution, the nonconvex constraint about main lobe is rewritten as a convex form, which leads to error. In order to reduce the error, an alternating optimization process is designed by introducing auxiliary phase. Since the auxiliary phase can only be approximately equal to the real phase, this approximation would bring error. To further improve the synthesis performance, we find the peak error point over main lobe and increase its weight in the iteration. Finally, numerical examples show the good performance of the proposed method in pattern synthesis.

\section{Problem Formulation}

Consider a dual-polarized conformal array composed of $N$ elements located on an arbitrary carrier surface, as shown in Figure 1. $O(x, y, z)$ and $O^{\prime}\left(x^{\prime}, y^{\prime}, z^{\prime}\right)$ represent the global Cartesian coordinate system and the local Cartesian coordinate system, respectively. Without loss of generality, it is assumed that the $\theta$-polarized and $\phi$-polarized components are generated by two individual feed points of each dualpolarized antenna.

The two orthogonal components of the array radiation pattern can be written as $\phi$-polarization pattern $E_{\phi}(\theta, \phi)$ and $\theta$-polarization pattern $E_{\theta}(\theta, \phi)$ :

$$
\begin{aligned}
E_{\theta}(\theta, \phi) & =\sum_{i=1}^{N} E_{i \theta}(\theta, \phi) \exp \left\{j k \mathbf{v}_{i}^{T} \mathbf{e}(\theta, \phi)\right\}, \\
& =\mathbf{a}^{T}(\theta, \phi) \mathbf{E}_{\theta}(\theta, \phi), \\
E_{\phi}(\theta, \phi) & =\sum_{i=1}^{N} E_{i \phi}(\theta, \phi) \exp \left\{j k \mathbf{v}_{i}^{T} \mathbf{e}(\theta, \phi)\right\}, \\
& =\mathbf{a}^{T}(\theta, \phi) \mathbf{E}_{\phi}(\theta, \phi),
\end{aligned}
$$

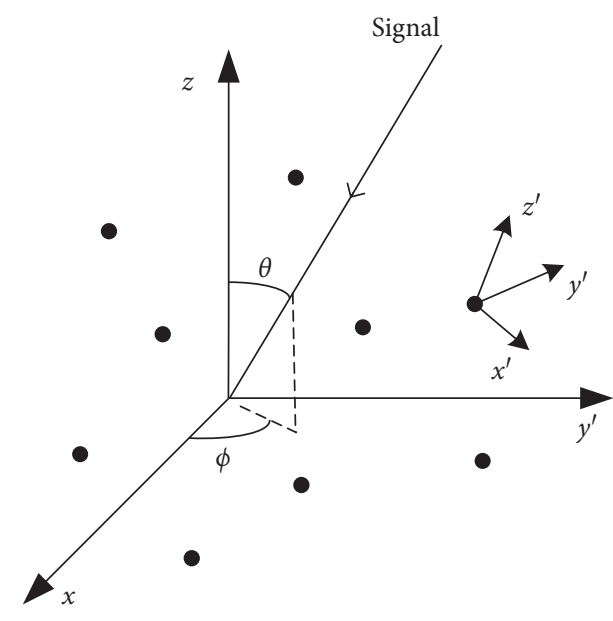

Figure 1: Coordinate systems.

where $E_{i \theta}(\theta, \phi)$ and $E_{i \phi}(\theta, \phi)$ are the contribution of the $i$ th element to the $\theta$-polarized and $\phi$-polarized components, respectively. $k=(2 \pi / \lambda)$ is the wavenumber, $\mathbf{v}_{i}=\left[x_{i}, y_{i}, z_{i}\right]^{T}$ is the position vector of the $i$ th element, $\mathbf{e}(\theta, \phi)=[\sin \theta \cos \phi$, $\sin \theta \sin \phi, \cos \theta]^{T}$ is the unit direction vector, $\{\bullet\}^{T}$ denotes the transposition, and $\mathbf{a}(\theta, \phi)=\left[e^{j k \mathbf{v}_{1}^{T} \mathbf{e}(\theta, \phi)}, \ldots, e^{j k \mathbf{v}_{N}^{T} \mathbf{e}(\theta, \phi)}\right]^{T}$ is the ideal steering vector of the array.

Note that the $\theta$-polarized and $\phi$-polarized components of each antenna are inconsistent because of the curved structure of conformal array. Therefore, they cannot be superimposed directly. The electromagnetic field excited by each element is decomposed and projected to the unified global coordinate system. And $E_{i \theta}(\theta, \phi)$ and $E_{i \phi}(\theta, \phi)$ can be obtained though some mathematical transformation, which are described in [19] in detail. The global to local Euler rotation matrix of the ith element is defined as $\mathbf{R}_{g l i}$, and $\mathbf{R}_{\mathrm{lg} i}$ denotes the local to global Euler rotation matrix of the $i$ th element. Then, $E_{i \theta}(\theta, \phi)$ and $E_{i \phi}(\theta, \phi)$ can be written as

$$
\begin{aligned}
& {\left[\begin{array}{c}
E_{i \theta}(\theta, \phi) \\
E_{i \phi}(\theta, \phi)
\end{array}\right]} \\
& \quad=\mathbf{T}_{c s}(\theta, \phi) \mathbf{R}_{\mathrm{lg} i} \mathrm{~T}_{s c i}\left(\theta_{i}^{\prime}, \phi_{i}^{\prime}\right)\left[\begin{array}{l}
w_{i \theta^{\prime}}^{*} g_{i \theta^{\prime}}\left(\theta_{i}^{\prime}, \phi_{i}^{\prime}\right) \\
w_{i \phi^{\prime}}^{*} g_{i \phi^{\prime}}\left(\theta_{i}^{\prime}, \phi_{i}^{\prime}\right)
\end{array}\right],
\end{aligned}
$$

where $\mathbf{T}_{c s}(\theta, \phi)$ is the transform matrix from Cartesian coordinate to spherical coordinate in global coordinate system and $\mathbf{T}_{\mathrm{sci}}\left(\theta_{i}^{\prime}, \phi_{i}^{\prime}\right)$ is the spherical coordinate to Cartesian coordinate transform matrix in local coordinate system. $\left(\theta_{i}^{\prime}, \phi_{i}^{\prime}\right)$ is the corresponding angle of $(\theta, \phi)$ in the $i$ th local coordinate and can been obtained though Euler rotation. $w_{i \theta^{\prime}}$ and $w_{i \phi^{\prime}}$ are excitation weights of two feed points, which control $\theta$-polarized and $\phi$-polarized patterns of the $i$ th element, respectively. $\{\bullet\}^{*}$ denotes conjugation. $g_{i \theta^{\prime}}\left(\theta_{i}^{\prime}, \phi_{i}^{\prime}\right)$ and $g_{i \phi^{\prime}}\left(\theta_{i}^{\prime}, \phi_{i}^{\prime}\right)$ are $\theta$-polarized and $\phi$-polarized patterns of the $i$ th element. Then, for all of the array elements, we have 


$$
\begin{aligned}
{\left[\begin{array}{c}
E_{1 \theta}(\theta, \phi) \\
E_{1 \phi}(\theta, \phi) \\
\vdots \\
E_{N \theta}(\theta, \phi) \\
E_{N \phi}(\theta, \phi)
\end{array}\right] } & =\left[\begin{array}{lll}
\mathbf{T}_{1}(\theta, \phi) & & \\
& \ddots & \\
& & \mathbf{T}_{N}(\theta, \phi)
\end{array}\right] \mathbf{G}(\theta, \phi) \mathbf{w}^{*} \\
& =\mathbf{T}(\theta, \phi) \mathbf{G}(\theta, \phi) \mathbf{w}^{*},
\end{aligned}
$$

where $\mathbf{T}_{i}(\theta, \phi)=\mathbf{T}_{c \mathcal{T}}(\theta, \phi) \mathbf{R}_{\mathrm{lg} i} \mathrm{~T}_{\mathrm{sci}}\left(\theta_{i}^{\prime}, \phi_{i}^{\prime}\right)$ and $\mathbf{w}=\left[w_{1 \theta^{\prime} \text {, }}\right.$ $\left.w_{1 \phi}, \ldots, w_{N \theta^{\prime}}, w_{N \phi^{\prime}}\right]^{T} . \mathbf{G}(\theta, \phi)=\operatorname{diag}\left[g_{1 \theta^{\prime}}\left(\theta_{1}^{\prime}, \phi_{1}^{\prime}\right), g_{1 \phi^{\prime}}\left(\theta_{1}^{\prime}\right.\right.$, $\left.\left.\phi_{1}^{\prime}\right), \ldots, g_{N \theta^{\prime}}\left(\theta_{N}^{\prime}, \phi_{N}^{\prime}\right), g_{N \phi^{\prime}}\left(\theta_{N}^{\prime}, \phi_{N}^{\prime}\right)\right]^{T}$. It should be noted that the size of $\mathbf{T}_{i}(\theta, \phi)$ is $2 \times 2$, which means that $E_{i \theta}$ or $E_{i \phi}$ depends on both $g_{i \theta^{\prime}}\left(\theta_{i}^{\prime}, \phi_{i}^{\prime}\right)$ and $g_{i \phi^{\prime}}\left(\theta_{i}^{\prime}, \phi_{i}^{\prime}\right)$. Through the above transformation, array pattern can be obtained by

$$
\begin{aligned}
{\left[\begin{array}{c}
E_{\theta}(\theta, \phi) \\
E_{\phi}(\theta, \phi)
\end{array}\right]^{T} } & =\left[\begin{array}{c}
\mathbf{a}^{T}(\theta, \phi) \mathbf{E}_{\theta}(\theta, \phi) \\
\mathbf{a}^{T}(\theta, \phi) \mathbf{E}_{\phi}(\theta, \phi)
\end{array}\right]^{T} \\
& =\left[\begin{array}{c}
E_{1 \theta}(\theta, \phi) \\
E_{1 \phi}(\theta, \phi) \\
\vdots \\
E_{N \theta}(\theta, \phi) \\
E_{N \phi}(\theta, \phi)
\end{array}\right]\left(\mathbf{a}^{T}(\theta, \phi) \otimes \mathbf{I}_{2}\right)^{T}, \\
& =\mathbf{w}^{H} \mathbf{G}(\theta, \phi) \mathbf{T}^{T}(\theta, \phi)\left(\mathbf{a}(\theta, \phi) \otimes \mathbf{I}_{2}\right) \\
& =\mathbf{w}^{H} \widetilde{\mathbf{a}}(\theta, \phi),
\end{aligned}
$$

where " $\otimes$ " denotes the Kronecker product, $\mathbf{I}_{2}$ is an identity matrix with the size of $2 \times 2$, and

$$
\widetilde{\mathbf{a}}(\theta, \phi)=\mathbf{G}(\theta, \phi) \mathbf{T}^{T}(\theta, \phi)\left(\mathbf{a}(\theta, \phi) \otimes \mathbf{I}_{2}\right) .
$$

It should be noted that $\widetilde{\mathbf{a}}(\theta, \phi)$ is a matrix of size $2 N \times 2$, which contains two steering vectors of both orthogonal polarization components. Although the above derivation is based on the linear polarization model, it can be easily extended to any generic elliptic polarization condition. For example, in the condition of circular polarization, the corresponding steering vector can be expressed as

$$
\widetilde{\mathbf{a}}_{c p}(\theta, \phi)=\mathbf{G}(\theta, \phi) \mathbf{T}^{T}(\theta, \phi)\left(\mathbf{a}(\theta, \phi) \otimes \mathbf{P}^{-T}\right),
$$

where

$$
\mathbf{P}=\left[\begin{array}{cc}
\frac{\sqrt{2}}{2} & -\frac{\sqrt{2}}{2} j \\
\frac{\sqrt{2}}{2} & \frac{\sqrt{2}}{2} j
\end{array}\right] .
$$

As long as we know the parameters of any polarization state, the steering vector of this polarization can be obtained.

The dual-polarized conformal array pattern synthesis problem aims at finding a weight vector $\mathbf{w}$ with the size of $2 N \times 1$ to achieve desired pattern shape. Specifically, the $\phi$-polarized component is assumed to be the copolarization component, and the requirements can be described as follows:

(i) The main lobe of beam $E_{\phi}(\theta, \phi)$ is close to the desired shape $\mathrm{d}(\theta, \phi)$ in the main lobe region $\Omega_{m}$.

(ii) The side lobe level of beam $E_{\phi}(\theta, \phi)$ is under a upper bound $\rho(\theta, \phi)$ in the side lobe region $\Omega_{s}$.

(iii) The cross-polarization $E_{\theta}(\theta, \phi)$ level cannot exceed the bound $q(\theta, \phi)$.

Therefore, this problem can be formulated as the following optimization problem:

$$
\begin{aligned}
& \min _{w} \quad \varepsilon, \\
& \text { s.t. } \quad|| E_{\phi}(\theta, \phi)|-\mathrm{d}(\theta, \phi)| \leq \varepsilon, \quad(\theta, \phi) \in \Omega_{m}, \\
& \quad\left|E_{\phi}(\theta, \phi)\right| \leq p(\theta, \phi), \quad(\theta, \phi) \in \Omega_{s}, \\
& \left|E_{\theta}(\theta, \phi)\right| \leq q(\theta, \phi), \quad(\theta, \phi) \in\left(\Omega_{m} \cup \Omega_{s}\right),
\end{aligned}
$$

where $\varepsilon$ denotes the maximum error between $E_{\phi}(\theta, \phi)$ and $\mathrm{d}(\theta, \phi)$ over $\Omega_{m}$. And through minimizing this error, we can obtain an array pattern approaching the desired one.

\section{Pattern Synthesis Method}

3.1. The Convex Optimization with Additional Auxiliary Phase. It is known that constraint (8b) is NP-hard because of the quadratic constraint. However, it should be noted that

$$
|| E_{\phi}(\theta, \phi)|-\mathrm{d}(\theta, \phi)| \leq\left|E_{\phi}(\theta, \phi)-e^{j \varphi(\theta, \phi)} \mathrm{d}(\theta, \phi)\right|,
$$

and if only $\varphi(\theta, \phi)=\angle E_{\phi}(\theta, \phi)$, the "=" in formula (9) holds.

Therefore, the constraint (8b) can be rewritten as

$$
\left|E_{\phi}(\theta, \phi)-e^{j \varphi(\theta, \phi)} \mathrm{d}(\theta, \phi)\right| \leq \varepsilon, \quad(\theta, \phi) \in \Omega_{m},
$$

where $\mathrm{d}(\theta, \phi)$ is often deemed to be real-valued and $\varphi(\theta, \phi) \in[-\pi, \pi]$ is defined as an auxiliary phase function.

In actual processing, there are many sampling angles over main lobe and side lobe. Thus each constraint in problem (8) contains a series of constraints. To ease the convex optimization programming, norm programming can be introduced. The constraint ( $8 \mathrm{~b})$ can be expressed as

$$
\left\|\mathbf{E}_{\phi m}-e^{j \Psi} \odot \mathbf{d}\right\|_{2} \leq \varepsilon,
$$

where $\mathbf{E}_{\phi m}=\left[E_{\phi}\left(\theta_{m_{1}}, \phi_{n_{1}}\right), \ldots, E_{\phi}\left(\theta_{m_{2}}, \phi_{n_{2}}\right)\right]$ denotes the discrete sampling of copolarization pattern in the sampling angles of main lobe region, $\Psi=\left[\varphi\left(\theta_{m_{1}}, \phi_{n_{1}}\right), \ldots, \varphi\left(\theta_{m_{2}}\right.\right.$, $\left.\left.\phi_{n_{2}}\right)\right], \mathbf{d}=\left[\mathrm{d}\left(\theta_{m_{1}}, \phi_{n_{1}}\right), \ldots, \mathrm{d}\left(\theta_{m_{2}}, \phi_{n_{2}}\right)\right]$, " $\odot$ " denotes the Hadamard product, and $\|\bullet\|_{2}$ denotes $L_{2}$ norm.

Similarly, other constraints in problem (8) can also be written as sampling vector form. The constraint (8c) can be expressed as

$$
\left\|\mathbf{E}_{\phi s}\right\|_{\infty} \leq \rho,
$$


where $\quad \mathbf{E}_{\phi s}=\left[E_{\phi}\left(\theta_{1}, \phi_{1}\right), \ldots, E_{\phi}\left(\theta_{m_{1}-1}, \phi_{n_{1}-1}\right), E_{\phi}\left(\theta_{m_{2}+1}\right.\right.$, $\left.\left.\phi_{n_{2}+1}\right), \ldots, E_{\phi}\left(\theta_{k_{1}}, \phi_{k_{2}}\right)\right]$ denotes the discrete sampling of copolarization pattern in the sampling angles of side lobe region.

The constraint (8d) can be expressed as

$$
\left\|\mathbf{E}_{\theta}\right\|_{\infty} \leq q
$$

where $\mathbf{E}_{\theta}=\left[E_{\theta}\left(\theta_{1}, \phi_{1}\right), \ldots, E_{\theta}\left(\theta_{k_{1}}, \phi_{k_{2}}\right)\right]$ denotes the discrete sampling of cross-polarization pattern and $\|\bullet\|_{\infty}$ denotes $L_{\infty}$ norm. $q$ and $\rho$ are both small real values.

Then problem (8) can be rewritten as

$$
\begin{array}{r}
\min _{w} \varepsilon, \\
\text { s.t. }\left\|\mathbf{E}_{\phi m}-e^{j \Psi} \odot \mathbf{d}\right\|_{2} \leq \varepsilon, \\
\left\|\mathbf{E}_{\phi s}\right\|_{\infty} \leq p, \\
\left\|\mathbf{E}_{\theta}\right\|_{\infty} \leq q .
\end{array}
$$

Observe that when $\varphi(\theta, \phi)$ is fixed, problem (14) is a jointly convex problem with respect to $\{\mathbf{w}, \varepsilon\}$. And if $\mathbf{w}$ is given, the phase $\varphi(\theta, \phi)$ can be obtained. However, before we obtain the optimal weight vector, we can only know an approximate phase. Therefore, an iterative process is introduced to make the approximate phase gradually approach the real one. Specifically, when $\mathbf{w}$ is given, the auxiliary phase $\varphi(\theta, \phi)$ can be updated as follows:

$$
\varphi^{(i+1)}(\theta, \phi)=\alpha \angle E_{\phi}^{(i+1)}(\theta, \phi)+(1-\alpha) \varphi^{(i)}(\theta, \phi),
$$

$\varphi^{(i+1)}(\theta, \phi)$ of next iteration is not only simply assigned by $\angle E_{\phi}^{(i+1)}(\theta, \phi)$, but also $\varphi^{(i)}(\theta, \phi)$ of last iteration. The parameter $\alpha$ determines the ratio of $\varphi^{(i)}(\theta, \phi)$ and $\angle E_{\phi}^{(i+1)}(\theta, \phi)$ in the process of obtaining $\varphi^{(i+1)}(\theta, \phi)$, and it is generally ranging from 0 to 1 . Therefore, an alternating optimization process can be applied.

3.2. The ICO Algorithm via Minimax Error. Although the algorithm described above can reduce error through additional auxiliary phase, it should be noted that the algorithm still has inevitable error because $\varphi(\theta, \phi)$ is not exactly equal to $\angle E_{\phi}(\theta, \phi)$. Thus it is necessary to find another way to further reduce the error and get smaller ripple of main lobe based on the above method.

For the constraint (14b), the "worst point" can be defined as

$$
(\theta, \phi)_{\text {worst }}^{(i)}=\arg \max _{(\theta, \phi) \in \Omega_{m}}\left(\left|E_{\phi}(\theta, \phi)\right|-\mathrm{d}(\theta, \phi)\right) .
$$

Compared with [20], the modulus of $E_{\phi}(\theta, \phi)$ is used because $\mathrm{d}(\theta, \phi)$ is real-valued. In the obtained pattern $\mathbf{E}_{\phi}$, the point which exceeds the bound $\mathbf{d}$ most in the main lobe region would be defined as the "worst point."
Then the point $(\theta, \phi)_{\text {worst }}$ would be added to the observation points of next iteration. The constraint (14b) can be expressed as

$$
\begin{aligned}
& \left\|\mathbf{E}_{\phi m}^{(i+1)}-e^{j \Psi^{(i+1)}} \odot \mathbf{d}^{(i+1)}\right\|_{2} \leq \mathcal{E}, \\
& \mathbf{E}_{\phi m}^{(i+1)}=\left[\mathbf{E}_{\phi m}^{(i)}, E_{\phi}(\theta, \phi)_{\text {worst }}^{(i)}\right]
\end{aligned}
$$

where $\Psi^{(i+1)}$ and $\mathbf{d}^{(i+1)}$ are similarly defined. After each iteration, the weight at the angle $(\theta, \phi)_{\text {worst }}$ would increase by "1" automatically. Therefore, the error between the obtained pattern and desired pattern can be reduced by accumulating the weights of some observation angles.

Through several iterations, the "worst point" may be at the same angle many times. Note that when the "worst point" are too concentrated, the weights of these points could be too large, which may cause the errors of other points to become larger and then may lead to worse result. In many cases, these worst points are most likely to be concentrated around the transitional region between the main lobe and the side lobe. Therefore, measures need to be carried out to avoid the above condition. Suppose that in the previous $i$ iterations, $L^{(i)}$ denotes the sum of errors in main lobe. Compare $L^{(i+1)}$ with $L^{(i)}$, and determine whether $\mathbf{w}$ will be updated. It can be specifically described as the following calculation procedure:

(1) Search for the "worst point" $(\theta, \phi)_{\text {worst }}$, and we can obtain $\Omega_{m}^{(i+1)}, \Omega_{m}^{(i+1)}=\left[\Omega_{m}^{(i)},(\theta, \phi)_{\text {worst }}^{(i)}\right]$.

(2) Calculate

$$
L^{(i)}=\left\|\mathbf{E}_{\phi m}-\mathbf{d}\right\|_{2} .
$$

(3) If $L^{(i+1)}<L^{(i)}$, update $\mathbf{w}$. Otherwise, $\mathbf{w}$ remains unchanged.

After each iteration, when the sum of errors in main lobe is reduced, w would be updated. Accordingly, when w remains unchanged, $L^{(i+1)}$ should be substituted by $L^{(i)}$. Through the above process, the convergence of the ICO algorithm can be guaranteed.

The initial value $L^{(0)}$ should be set reasonably because it has an impact on the convergence of the algorithm. In the preparation stage, we solve problem (14) without auxiliary phase function by convex optimization toolbox. $\angle E_{\phi}^{(0)}(\theta, \phi)$ can be obtained. Then the sum of errors over main lobe is set as $L^{(0)}$. And $\angle E_{\phi}^{(0)}(\theta, \phi)$ can be used as $\varphi^{(0)}(\theta, \phi)$. The flow chart of the proposed algorithm is shown in Figure 2, and the problem (14) can be solved by convex optimization solver in the toolbox, such as the CVX [21].

\section{Synthesis Examples}

The numerical examples of pattern synthesis are presented here to verify the effectiveness of the proposed method. Patch antenna is one of the most frequently used antennas in conformal array. In the following simulation, the lowest order circular patch antenna model [22] is adopted, and its pattern can be written as follows: 


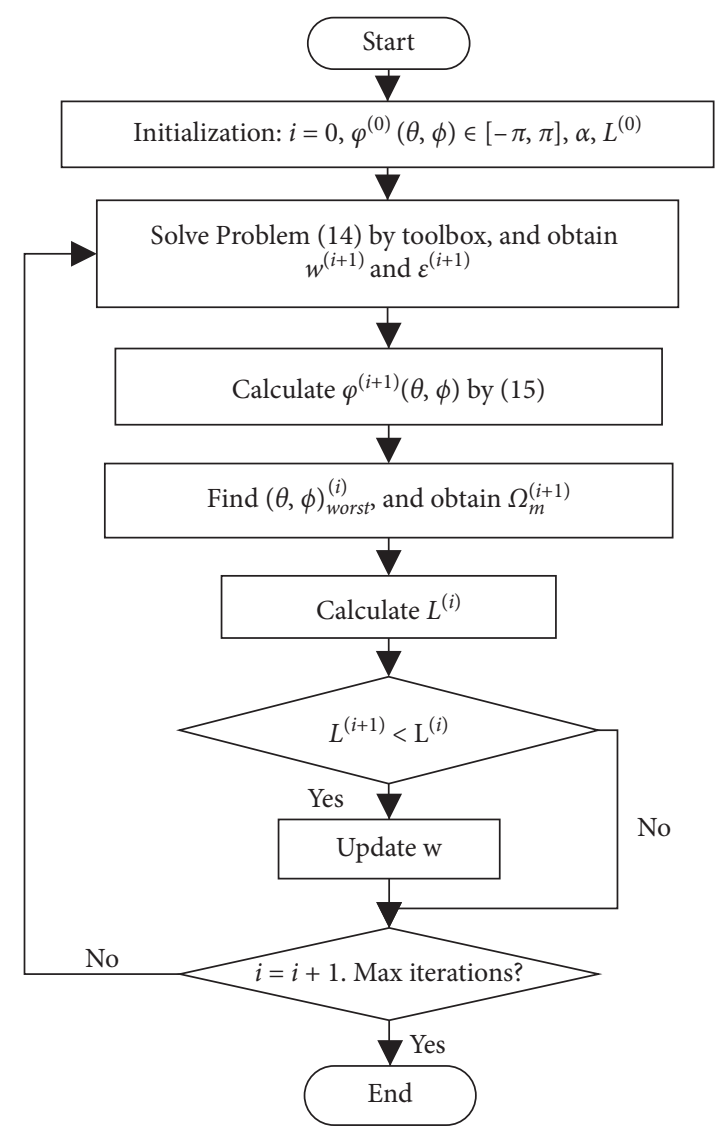

Figure 2: Algorithm flow chart.

$$
\begin{cases}g_{\theta}\left(\theta^{\prime}, \phi^{\prime}\right)=\left[J_{2}\left(\frac{\pi d}{\lambda} \sin \theta^{\prime}\right)-J_{0}\left(\frac{\pi d}{\lambda} \sin \theta^{\prime}\right)\right] \cdot\left[\cos \phi^{\prime}-j \sin \phi^{\prime}\right], & \theta^{\prime} \in\left[0, \frac{\pi}{2}\right], \\ g_{\phi}\left(\theta^{\prime}, \phi^{\prime}\right)=\left[J_{2}\left(\frac{\pi d}{\lambda} \frac{\sin \theta^{\prime}}{\lambda}\right)+J_{0}\left(\frac{\pi d}{\lambda} \sin \theta^{\prime}\right)\right] \cdot \cos \theta^{\prime}\left[\sin \phi^{\prime}-j \cos \phi^{\prime}\right], & \theta^{\prime} \in\left[0, \frac{\pi}{2}\right], \\ g_{\theta}\left(\theta^{\prime}, \phi^{\prime}\right)=g_{\phi}\left(\theta^{\prime}, \phi^{\prime}\right)=0, & \text { Otherwise, }\end{cases}
$$

where $J_{2}$ and $J_{0}$ are the first kind of second-order and zeroorder Bessel functions, respectively. A convex optimization method via linear programming (LP) [23] is applied for comparison, where the upper bound and lower bound are set. And the upper bound is defined as the desired pattern in the proposed method. Two typical conformal arrays with half-wavelength-spaced elements are used to verify the effectiveness of the algorithm in the following.

4.1. Semicircular Arc Array. In this subsection, pattern synthesis is carried out based on a semicircular arc array as shown in Figure 3. The goal of the first example is to achieve a flat-top beam of $\phi$-polarization components. With the fixed elevation angle $\theta=90^{\circ}$, the main lobe region of desired pattern is $\phi \in\left[-30^{\circ}, 30^{\circ}\right]$, and the side lobe region is $\phi \in\left[-90^{\circ},-30^{\circ}\right) \cup\left(30^{\circ}, 90^{\circ}\right]$. The cross-polarization level is optimized in the whole observation region. And the value of $\alpha$ is 0.5 in this experiment. Figure 4 shows the synthesis result of copolarization component and cross-polarization component. The initial pattern is obtained by solving convex problem without auxiliary phase, as described in setting the initial values. In the case of achieving similar side lobe level $-40 \mathrm{~dB}$ and cross-polarization level $-50 \mathrm{~dB}$, the proposed ICO algorithm and LP method perform better than the initial pattern on main lobe shaping. By the ICO method, the ripple of gain level in main lobe is less than that of the LP method. The result shows that the optimization performance of the ICO algorithm is improved. And the corresponding elements weights are shown in Figures 5 and 6.

After 100 iterations, the count of the "worst points" in $\Omega_{m}^{(i+1)}$ is plotted in Figure 7 . Note that the sum of errors in main lobe stops reducing through 28 iterations. Therefore, there are only 28 "worst points" in $\Omega_{m}^{(i+1)}$. It can be seen that 


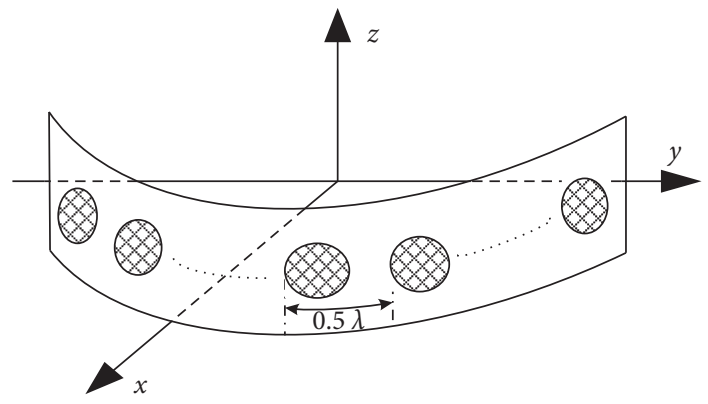

FIgURE 3: Schematic view of the conformal array with 30 elements.

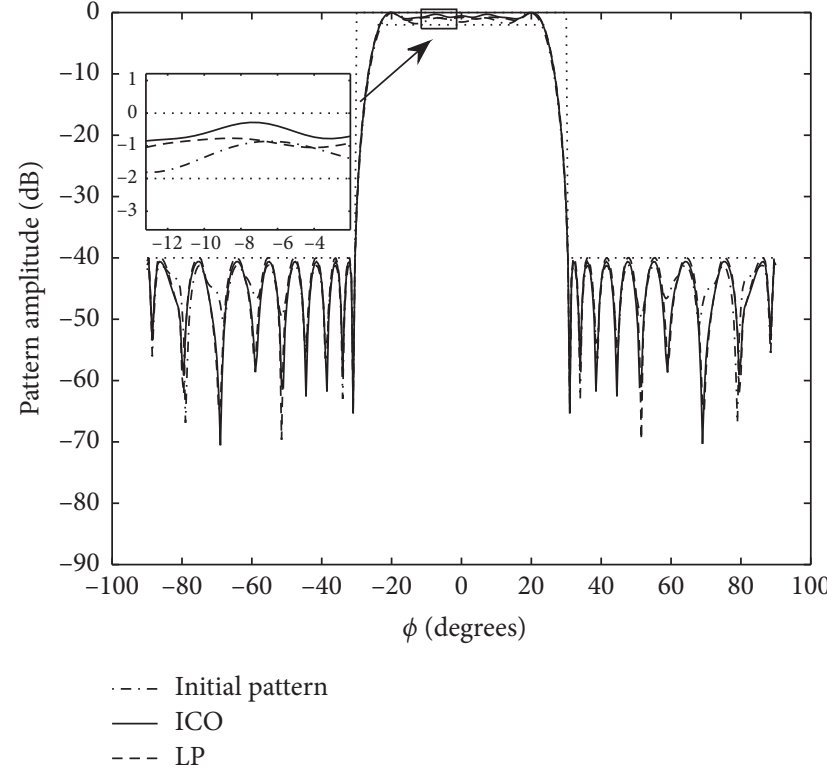

(a)

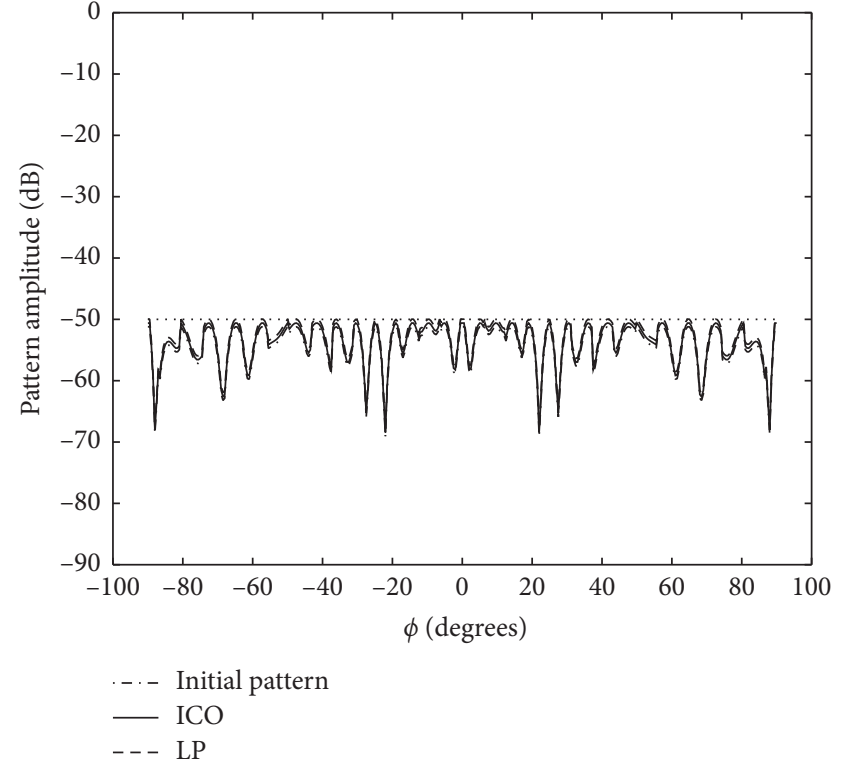

(b)

Figure 4: Flat-top-shaped beam synthesis of a 30-element array. (a) Copolarization component $\mathbf{E}_{\phi}$ at $\theta=90^{\circ}$. (b) Cross-polarization component $\mathbf{E}_{\theta}$ at $\theta=90^{\circ}$.

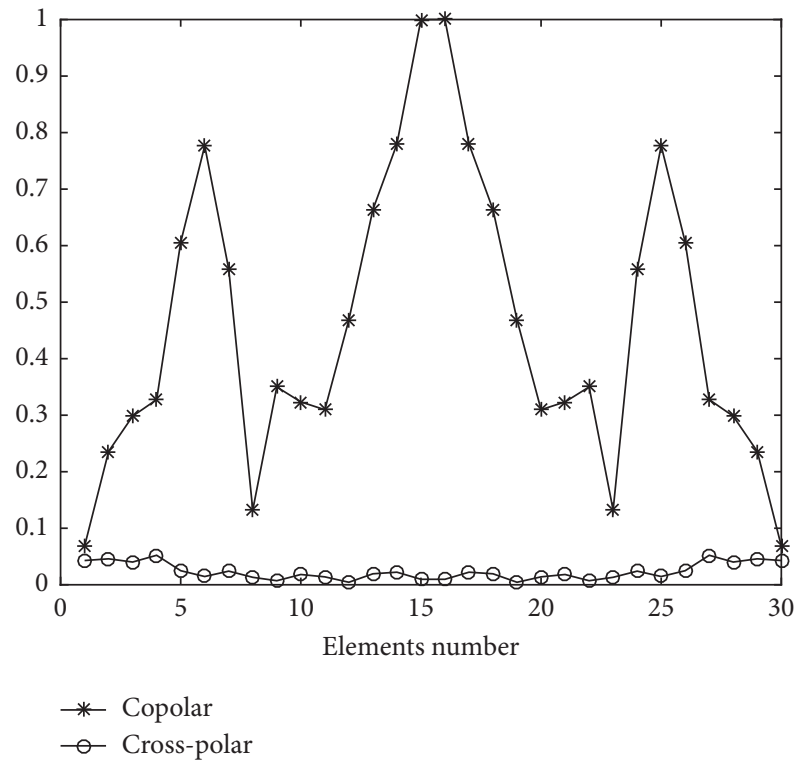

FIGURE 5: Normalized amplitudes of the elements weights.

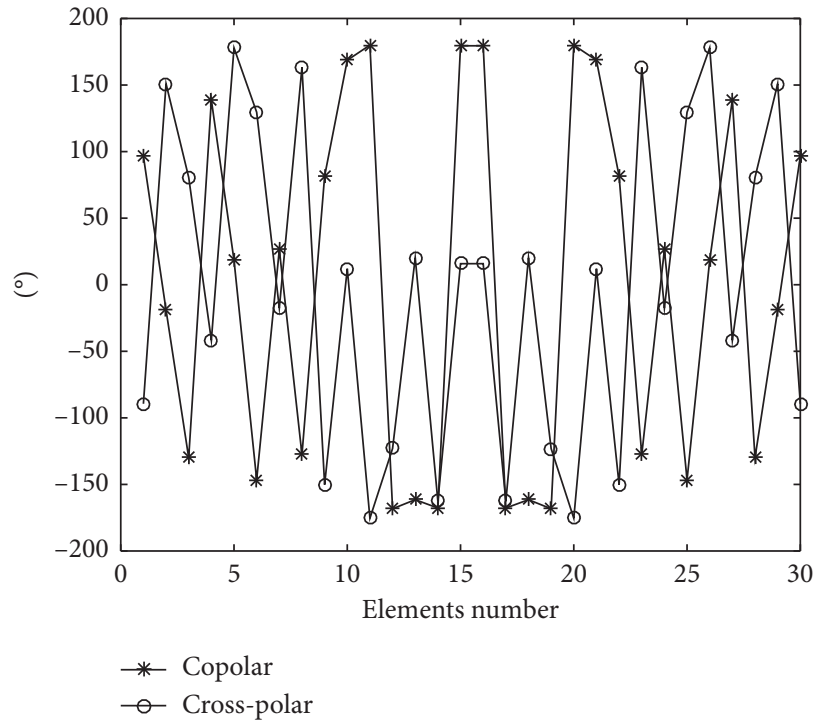

Figure 6: Phases of the elements weights. 


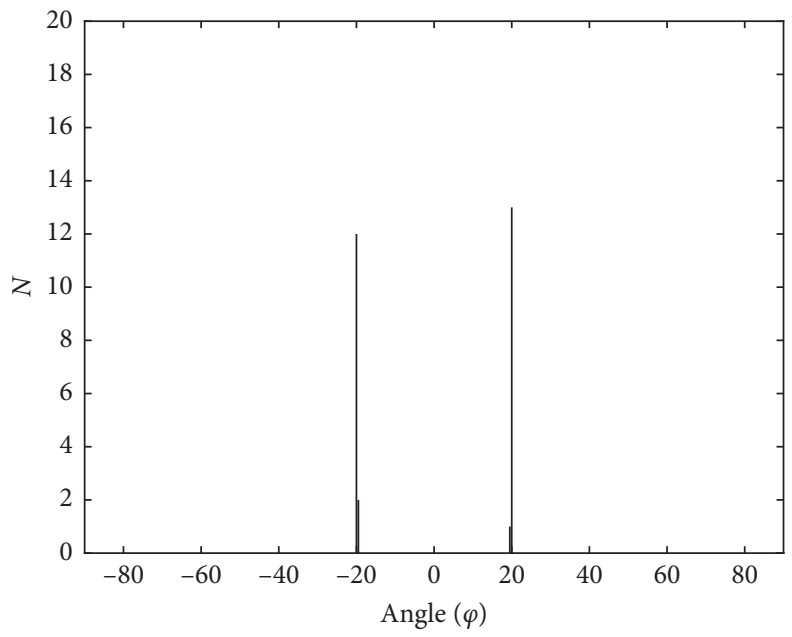

Figure 7: Count of the "worst point" (iterations=100).

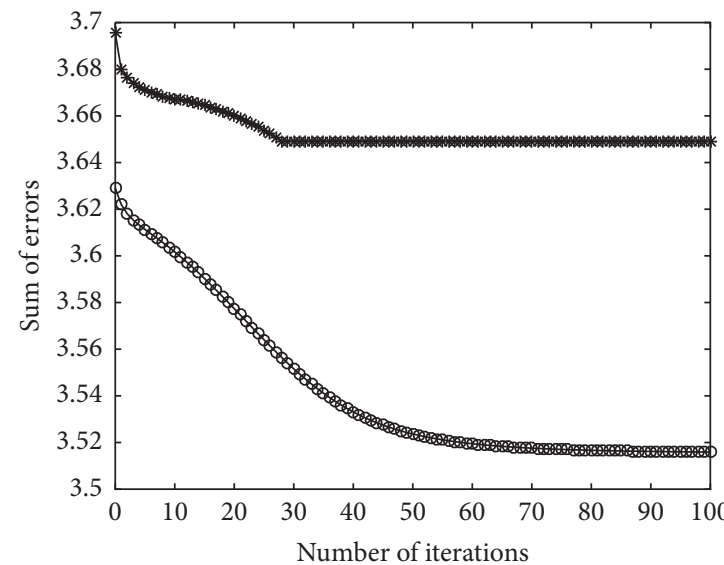

$\rightarrow$ Proposed method

$\rightarrow$ LP

FIGURE 8: $L^{(i)}$ versus the number of iterations.

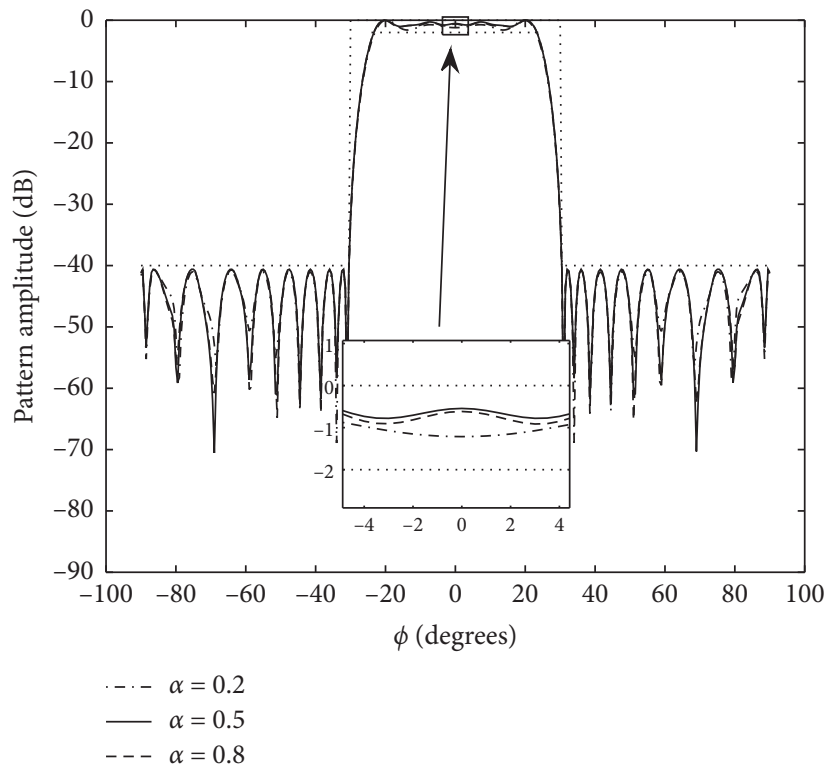

Figure 9: Copolarization components with different $\alpha$. 


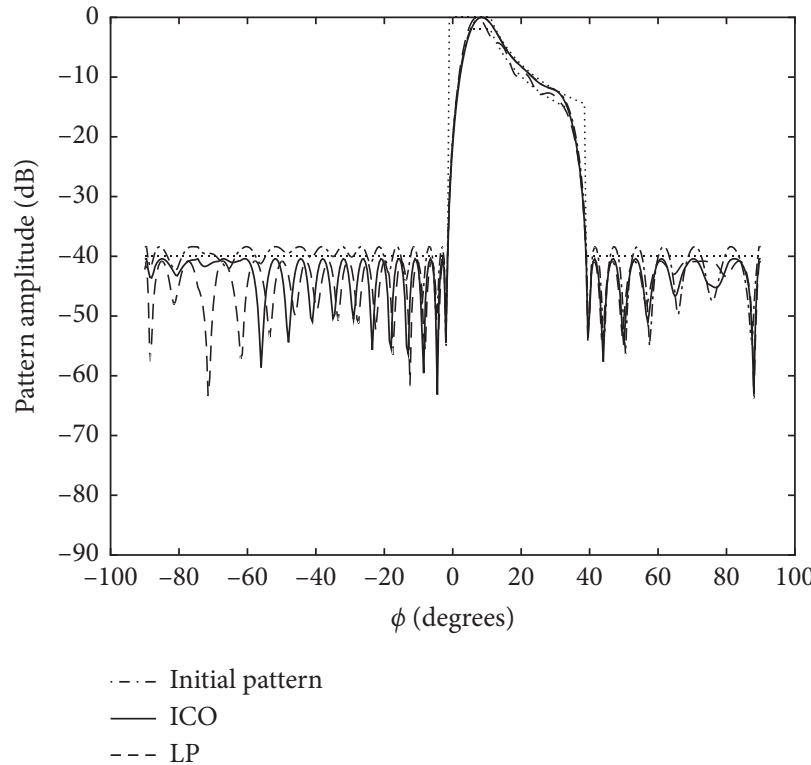

(a)

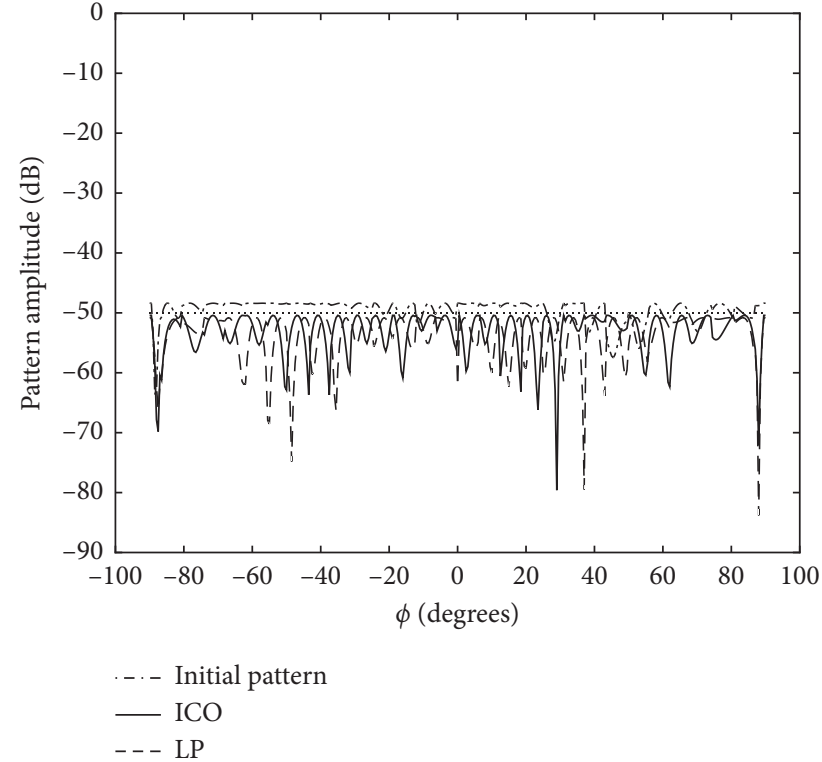

(b)

FIgURE 10: Cosecant-square-shaped beam synthesis. (a) Copolarization component $\mathbf{E}_{\phi}$ at $\theta=90^{\circ}$. (b) Cross-polarization component $\mathbf{E}_{\theta}$ at $\theta=90^{\circ}$.

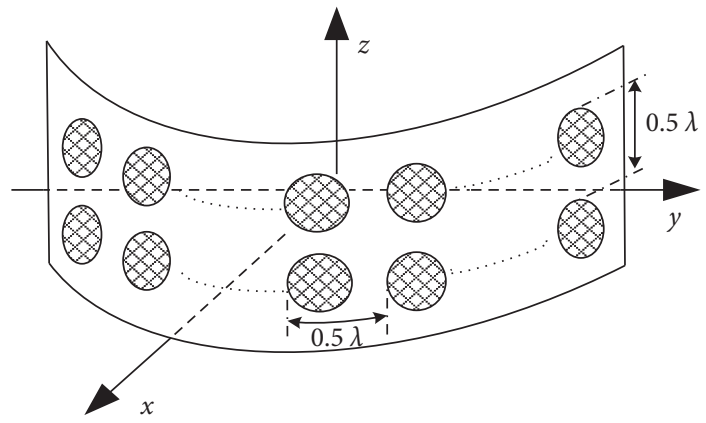

FIGURE 11: Schematic view of the conformal array with $2 \times 40$ elements.

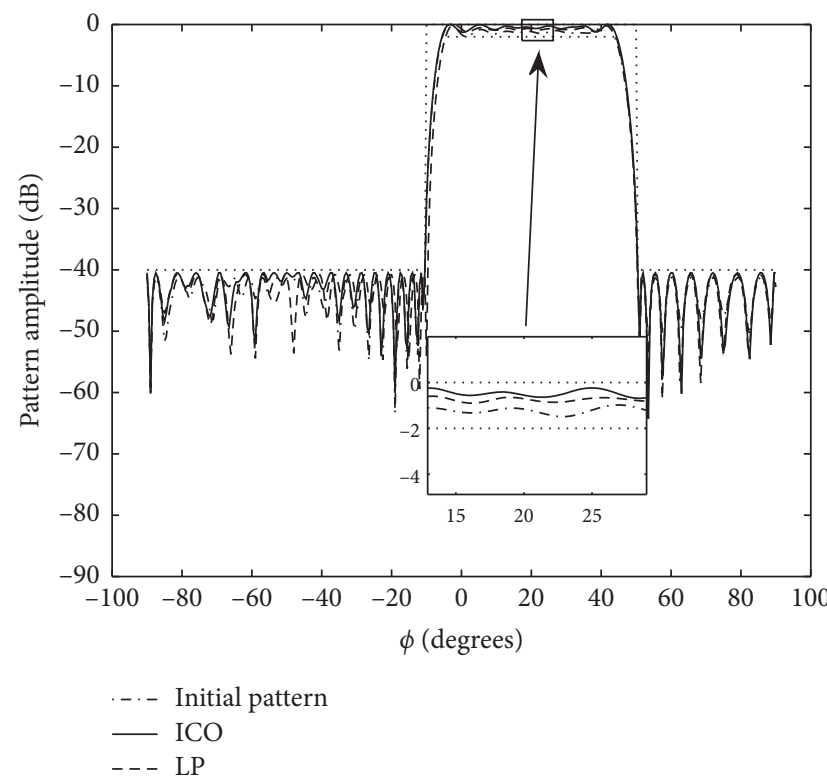

(a)

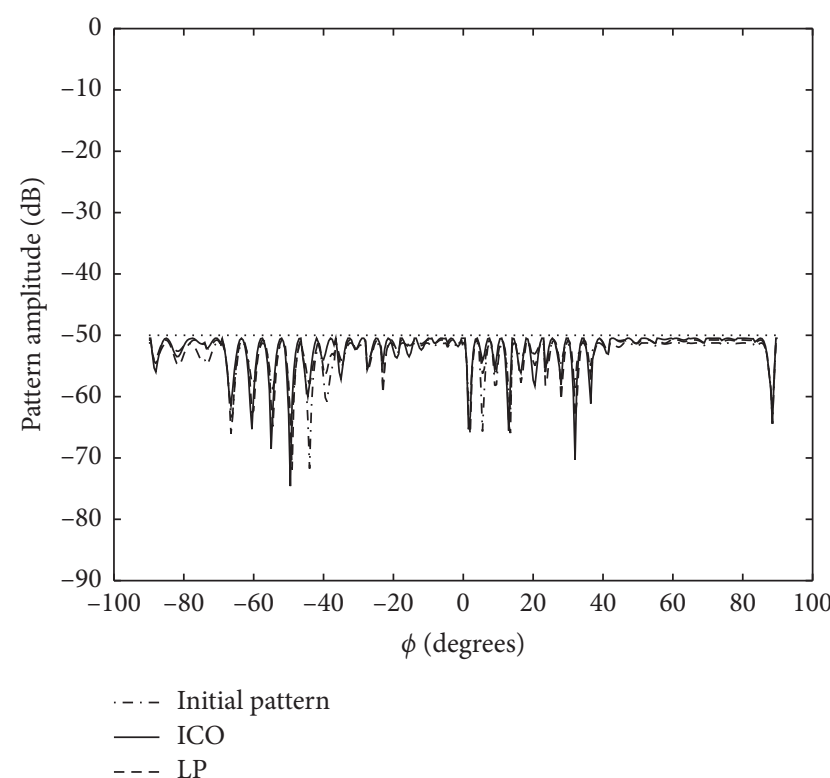

(b)

Figure 12: Flat-top-shaped beam synthesis of a $2 \times 40$-element array. (a) Copolarization component $\mathbf{E}_{\phi}$ at $\theta=90^{\circ}$. (b) Cross-polarization component $\mathbf{E}_{\theta}$ at $\theta=90^{\circ}$. 
the "worst points" are centered around $\pm 20^{\circ}$. The error caused by the transition region is relatively large. Thus the result is reasonable.

The sum of errors in main lobe $L^{(i)}$ is defined as an index related to convergence. Since $L^{(i)}$ is not involved in the LP algorithm, it is uniformly calculated by (18). According to the first experiment, the main lobe region is $\phi \in\left[-30^{\circ}, 30^{\circ}\right]$, and so is the calculation range of (18). And it should be noted that due to different desired patterns, the sum of LP algorithm $\left(\mathbf{d}=\left(\mathbf{d}_{u p}+\mathbf{d}_{\text {low }}\right) / 2\right)$ error appears smaller than that of the proposed ICO method $\left(\mathbf{d}=\mathbf{d}_{\mathrm{up}}\right)$, where $\mathbf{d}_{\mathrm{up}}$ denotes the upper bound and $\mathbf{d}_{\text {low }}$ denotes the lower bound. The convergence curve can be seen in Figure 8. After 28 iterations, the ICO algorithm converges, but LP method does not. Therefore, the proposed ICO method performs better on convergence.

Further experiments are carried out to analyse the performance of the proposed method with different $\alpha$, as shown in Figure 9. The results show that $\alpha$ has an effect on the ripple of main lobe. If we want to get the optimal solution, it is necessary to select that by trial and error according to actual requirements.

The second example is to shape a cosecant-square beam over main lobe region defined as $\left[0^{\circ}, 40^{\circ}\right]$ with the array shown in Figure 3. Simultaneously, the side lobe level and cross-polarization level should be optimized. Figure 10 shows the optimized patterns with $\alpha=0.2$. When using the proposed ICO method, the main lobe is similar to the initial pattern. But side lobe level and cross-polarization level are about $2 \mathrm{~dB}$ lower than those of initial pattern. Although the LP method can achieve the similar side lobe level and cross-polarization level with the proposed algorithm, its ripple of main lobe is larger. To sum up, the proposed algorithm has a better overall performance on cosecant-square beam shaping, side lobe level, and cross-polarization level suppression.

4.2. Semicylindrical Array. In this subsection, the synthesis of flat-top beam centered at $20^{\circ}$ with a semicylindrical array of $2 \times 40$ elements is considered, as shown in Figure 11. With the fixed elevation angle $\theta=90^{\circ}$, the main lobe and side lobe regions of desired pattern are $\phi \in\left[-10^{\circ}, 50^{\circ}\right]$ and $\phi \in\left[-90^{\circ}\right.$, $\left.-10^{\circ}\right) \cup\left(50^{\circ}, 90^{\circ}\right]$, respectively. The cross-polarization component is suppressed in the whole observation region. And the value of $\alpha$ is 0.7. Figure 12 shows that the ICO and LP methods can achieve similar side lobe level $-40 \mathrm{~dB}$ and cross-polarization level $-50 \mathrm{~dB}$ with the initial pattern. The proposed ICO and LP methods perform better than the initial pattern on main lobe shaping. In addition, the ICO method can achieve pattern with less ripple of main lobe than LP method. In the case of using different arrays and setting main lobe centered at different directions, the above result shows the good adaptability of the proposed ICO method.

Considering all the experiment results, the comparative algorithm can also have a good performance, but the proposed method performs better on ripple of main lobe and cross-polarization level. Thus the algorithm improvement achieves a good effect.

\section{Conclusion}

This paper proposes an ICO algorithm to synthesize pattern for dual-polarized conformal arrays. A series of constraints are formulated as a joint optimization problem. In the process of solving this nonconvex problem, the convex form for the constraint of main lobe leads to error. Then an auxiliary phase function is introduced to reduce this error through an alternating optimization process. But the auxiliary phase cannot be completely equal to the real phase, and the error occurs in the main lobe. To get better performance, we find the "worst point" with the peak error in main lobe region, and then the performance of main lobe shaping is improved by increasing the weight at "worst point" iteratively. Finally, numerical experiments are carried out, and results show the well convergence and excellent capability of beam shaping of the proposed ICO method.

\section{Data Availability}

The image data used to support the findings of this study are available from the corresponding author upon request.

\section{Conflicts of Interest}

The authors declare that there are no conflicts of interest regarding the publication of this paper.

\section{References}

[1] T. E. Morton and K. M. Pasala, "Pattern synthesis of conformal arrays for airborne vehicles," in Proceedings of the IEEE Aerospace Conference, pp. 1030-1039, Big Sky, MT, USA, March 2004.

[2] J. O. Yang, Q. R. Yuan, F. Yang, H. J. Zhou, Z. P. Nie, and Z. Q. Zhao, "Synthesis of conformal phased array with improved NSGA-II algorithm," IEEE Transactions on Antennas and Propagation, vol. 57, no. 12, pp. 4006-4009, 2009.

[3] J. A. Ferreira and F. Ares, "Pattern synthesis of conformal arrays by the simulated annealing technique," Electronics Letters, vol. 33, no. 14, pp. 1187-1189, 1997.

[4] M. Li, X. Wang, Y. Li, and H. Dai, "Polarization beam pattern synthesis based on particle swarm optimization," IEICE Electronics Express, vol. 9, no. 21, pp. 1648-1653, 2012.

[5] R. Li, L. Xu, Y. Yin et al., "Low-cross-polarisation synthesis of conformal antenna arrays using a balanced dynamic differential evolution algorithm," IET Microwaves Antennas and Propagation, vol. 11, no. 13, pp. 1855-1862, 2017.

[6] L. I. Vaskelainen, "Iterative least-squares synthesis methods for conformal array antennas with optimized polarization and frequency properties," IEEE Transactions on Antennas and Propagation, vol. 45, no. 7, pp. 1179-1185, 1997.

[7] L. I. Vaskelainen, "Constrained least-squares optimization in conformal array antenna synthesis," IEEE Transactions on Antennas and Propagation, vol. 55, no. 3, pp. 859-867, 2007.

[8] S.-L. Chen, P.-Y. Qin, Y. J. Guo, Y. Liu, and P. You, "Generalized 2-D numerical pattern synthesis algorithm for low cross polarization and low sidelobe synthesis," IEEE Antennas and Wireless Propagation Letters, vol. 16, pp. 2578-2581, 2017.

[9] F. Zhao, S. Chai, H. Qi et al., "Hybrid alternate projection algorithm and its application for practical conformal array 
pattern synthesis," Journal of Systems Engineering and Electronics, vol. 23, no. 5, pp. 625-632, 2012.

[10] M. Comisso and R. Vescovo, "Fast co-polar and cross-polar $3 \mathrm{D}$ pattern synthesis with dynamic range ratio reduction for conformal antenna arrays," IEEE Transactions on Antennas and Propagation, vol. 61, no. 2, pp. 614-626, 2013.

[11] H. Lebret and S. Boyd, "Antenna array pattern synthesis via convex optimization," IEEE Transactions on Signal Processing, vol. 45, no. 3, pp. 526-532, 1997.

[12] K. M. Tsui and S. C. Chan, "Pattern synthesis of narrowband conformal arrays using iterative second-order cone programming," IEEE Transactions on Antennas and Propagation, vol. 58, no. 6, pp. 1959-1970, 2010.

[13] M. Li, Y. Chang, Y. Li, J. Dong, and X. Wang, "Optimal polarised pattern synthesis of wideband arrays via convex optimisation," IET Microwaves, Antennas \& Propagation, vol. 7, no. 15, pp. 1228-1237, 2013.

[14] B. Fuchs and J. J. Fuchs, "Optimal polarization synthesis of arbitrary arrays with focused power pattern," IEEE Transactions on Antennas and Propagation, vol. 59, no. 12, pp. 4512-4519, 2011.

[15] Y. Liu, J. Bai, K. D. Xu et al., "Linearly polarized shaped power pattern synthesis with sidelobe and cross-polarization control by using semidefinite relaxation," IEEE Transactions on Antennas and Propagation, vol. 66, no. 6, pp. 3207-3212, 2018.

[16] A. Massa, P. Rocca, and G. Oliveri, "Compressive sensing in electromagnetics - a review," IEEE Antennas and Propagation Magazine, vol. 57, no. 1, pp. 224-238, 2015.

[17] P. Cao, J. S. Thompson, H. Haas et al., "Constant modulus shaped beam synthesis via convex relaxation," IEEE Antennas and Wireless Propagation Letters, vol. 16, pp. 617-620, 2017.

[18] W. Hu, X. Wang, Y. Li, and S. Xiao, "Synthesis of conformal arrays with matched dual-polarized patterns," IEEE Antennas and Wireless Propagation Letters, vol. 15, pp. 1341-1344, 2016.

[19] C. Liu, Z. Ding, and X. Liu, "Pattern synthesis for conformal arrays with dual polarized antenna elements," in Proceedings of the International Congress on Image and Signal Processing, pp. 968-973, Dalian, China, October 2014.

[20] F. Wang, R. Yang, and C. Frank, "A new algorithm for array pattern synthesis using the recursive least squares method," IEEE Signal Processing Letters, vol. 10, no. 8, pp. 235-238, 2003.

[21] CVX Research, Inc., "CVX: MATLAB software for disciplined convex programming, Version 2.1," Dec. 2018 [Online]. Available: http://cvxr.com/cvx.

[22] J. R. James, P. S. Hall, and C. Wood, Microstrip Antenna Theory and Design, Peter Peregrinus, New York, NY, USA, 1981.

[23] B. Fuchs, "Shaped beam synthesis of arbitrary arrays via linear programming," IEEE Antennas and Wireless Propagation Letters, vol. 9, pp. 481-484, 2010. 